\title{
A NEW POSITION-SENSITIVE PHOTON DETECTOR BASED ON AN IMAGING SILICON PIXEL ARRAY (ISPA-Tube) ${ }^{*}$
}

T. Gys, C. D’Ambrosio, H. Leutz, D. Piedigrossi, D. Puertolas

CERN, Geneva, Switzerland

\begin{abstract}
A new position-sensitive photon detector has been developed and operated for the first time. It consists of a photocathode, evaporated on an optical fibre window, confronted at $30 \mathrm{~mm}$ distance with a silicon chip containing 1024 pixels of $75 \mu \mathrm{m} \times 500 \mu \mathrm{m}$ edges and bump-bonded to their individual readout electronics. This ISPA-tube is particularly suited to particle tracking with scintillating fibres (particle- and nuclear physics, medicine, biology, etc.) and to position-sensitive photon counting from low intensity light sources (astronomy, etc.). Examples for both kinds of application are displayed.
\end{abstract}

Submitted to Nucl. Instr. and Methods

\footnotetext{
${ }^{*}$ The work reported here is part of the LAA Project.
} 
The commonly adopted readout techniques for light signals from scintillating fibres are image intensifiers, photomultipliers and photodiodes. High spatial resolution for particle tracks is only provided via image intensifiers, which amplify the signals between their light detecting photocathodes and their light-emitting phosphor screens. After several stages of image intensifiers the amplified light signals show sufficient signal-to-noise ratio to be finally converted into electronic ones by CCD chips.

To avoid this technically complicated and rather bulky and costly solution, which degrades the original two-track resolution, reduces both the hit densities of the scintillating fibres and the signal-to-noise ratio, we developed within the RD-7 collaboration an Imaging Silicon Pixel Array (ISPA)-tube [1,2,3]. In a simple description, the ISPA-tube replaces the phosphor screen of an image intensifier by an anode chip mounted inside its vacuum-sealed envelope. Our first prototype ${ }^{\# 1}$ works with a $8 \mathrm{~mm} \times 4.8 \mathrm{~mm}$ silicon chip $\# 2$ (developed by the RD-19 collaboration at CERN) containing 1024 rectangular pixels with edges of $500 \mu \mathrm{m} \times 75 \mu \mathrm{m}$. Each silicon pixel is bonded with $\mathrm{Pb}-\mathrm{Sn}$ solder bumps of $38 \mu \mathrm{m}$ diameter and $13 \mu \mathrm{m}$ height to its proper electronic front-end channel, which contains preamplifier, comparator with adjustable threshold, delay line, coincidence logic and memory element $[4,5]^{\sharp 3}$. The pixel response to photoelectrons is thus binary. The entire arrangement is vacuum-sealed inside the ISPA-tube, the bump-bonding of the individual pixel electronics minimizes the noise and the line-parallel readout reduces the number of connections leading through the tube's envelope to only 34 instead of more than a thousand.

The ISPA-tube should not be confounded with another development, where Electron-Bombarded Charge Coupled Devices (EBCCD) are used instead of a phosphor screen. Their rather poor electronic capabilities prevent them from being triggered and their readout time is about 20 milliseconds. Their signal-to-noise ratio is limited and because of these drawbacks they must be used together with at least one conventional image intensifier. In contrast, the ISPA-tube is readout about thousand times faster in a line-parallel mode $\# 4$, has an excellent signal-to-noise ratio, can be triggered (via its pixel delay lines) and can be gated (via its strobed output).

After careful adjustment of the principal chip parameters with calibrated LED flashes (430 nm, $20 \mathrm{~ns}$ duration) we installed the following arrangement (Fig. 1) to track cosmic muons: a ribbon ( $10 \mathrm{~mm}$ wide, $5 \mathrm{~mm}$ thick, $200 \mathrm{~mm}$ long) containing eight plastic fibre bundles was closely attached to the optical fibre window $(5 \mathrm{~mm}$ thick, $10 \mu \mathrm{m}$ fibre diameter, numerical aperture $\mathrm{NA}=1$ ) of the ISPA-tube. Each bundle $\# 5$ is of square cross-section with $2.5 \mathrm{~mm}$ edges [6]. It contains 1600 coherently aligned individual fibres of $60 \mu \mathrm{m}$ diameter, fused together during repeated drawing procedures. Each fibre $(\mathrm{NA}=0.7)$ is of hexagonal shape and separated from its next neighbour by a double cladding [7] of standard PMMA $(n=1.49)$ and fluorinated polymethacrylate

\#1 Assembled and manufactured by BV Delft Electronische Producten (DEP), NL-9300 AB Roden, The Netherlands. The chip was wire bonded at U.C.I. Microélectronique, F-91946 Les Ulis, France.

\#2 In front of the chip a diaphragm limits the active chip surface to $7 \mathrm{~mm} \times 4 \mathrm{~mm}$.

\#3 We are indebted to E. H. M. Heijne, who kindly provided us with the silicon chip. The CERN-ECP Microelectronics group designed it and supervised its manufacturing at different places: Canberra Semiconductor NV, B-2430 Olen, Belgium (silicon pixel array); GEC-Marconi Materials Technology Ltd, Caswell Towchester NN 128 EQ, United Kingdom (bump bonding); Smart Silicon System SA, $\mathrm{CH}-1012$ Lausanne, Switzerland (electronics).

\#4 The 64 columns of 16 pixels can be readout at a frequency of 5 to $10 \mathrm{MHz}$, resulting in readout times of 6 to $12 \mu \mathrm{s}$.

\#5 Produced by Kuraray Co. Ltd, Tokyo, Japan. 
$(n=1.42)$. All fibres are doped with the 1-component scintillator PMP420 [8,9]. The hodoscope to allow for vertical cosmic triggers is indicated in the same figure.

The present ISPA-tube is a single-loop magnetic-focussed image intensifier $\# 6$. With its photocathode-anode gap of $30 \mathrm{~mm}$ and an acceleration voltage of $20 \mathrm{kV}$, the focussing condition is achieved by a 500 Gauss solenoidal field, which is the field we have used for the tests.

To track cosmic muons, the ISPA-tube was oriented in such a way that the $500 \mu \mathrm{m}$ edges of the pixels were vertical. For the spatial precision $\sigma$ this results in:

$$
\sigma^{2}=\mathrm{f}^{2}+\mathrm{p}^{2}+\mathrm{k}^{2}
$$

with $\mathrm{f}=60 / \sqrt{ } 9 \mu \mathrm{m}$ and $\mathrm{p}=75 / \sqrt{ } 12 \mu \mathrm{m}$ indicating the fibre- and the short pixel precisions, respectively. Finally, $\mathrm{k}$ indicates the term to be attributed to the magnetic focussing conditions of the ISPA-tube, to the alignment precision of the fibre bundles and to the range of the produced delta rays in the bundles. Typical tracks from cosmic muons traversing the fibre ribbon are shown in Fig. 2. The oblong pixel dimensions are adapted to the principal orientation of the particle tracks: the $500 \mu \mathrm{m}$ pixel edges go with the expected particle direction according to the tracker layout proposed in ref. [10] and supposed for the track reconstruction in ref. [11], whereas the $75 \mu \mathrm{m}$ edge allows for good spatial precision and two-track resolution. The spatial precision (standard deviation $\sigma$ of the residuals) of a few dozens of cosmic muon-tracks amounts to $\sim 40 \mu \mathrm{m}$, which yields for the term $\mathrm{k}$ a value of $\sim 27 \mu \mathrm{m}$. This corresponds to a two-track resolution [6] of FWHM $=2.4 \sigma=96 \mu \mathrm{m}$. The measured values for the precisions of the track centerof-gravity and of the track direction (which correspond to the precisions of the origin and direction of the generated microvectors) are respectively $10 \mu \mathrm{m}$ and $5 \mathrm{mrad}[11,12]$.

A photoelectron spectrum is displayed in Fig. 3. It was taken with LED flashes guided through the fibre ribbon onto the photocathode of the ISPA-tube and shows an average number of 4.22 photoelectrons. Because of the binary pixel readout, the photoelectrons are accumulated only at integer numbers (number of hit pixels per event). In particular, the backscattered photoelectrons (18\%) are not counted. This is in contrast to the analog spectra we measured with the HPMT-tube $[13,14]$, where the energy deposit of backscattered photoelectrons builds up a continuum below the discrete photoelectron peaks (Fig. 3 in ref. [14]). All counts between pedestal and 12 photoelectrons fit exactly the Poisson distribution for $\langle n\rangle=4.22$ photoelectrons. The tail between 12 and 20 photoelectrons is caused by instabilities of the LED flash intensities. Above 20 photoelectrons we see the noise contribution ( $\sim 5$ counts), which does not affect the counting rates of the photoelectrons. Further tests with this ISPA-tube are foreseen in a SPS $\pi^{-}$-beam at CERN. This will allow us to fully characterize the device.

To conclude, we have demonstrated for the first time the operation of a photoelectronic image intensifier with an integrated silicon pixel array (ISPA-tube). This device is sensitive to single photons and their original positions and we have successfully tracked cosmic muons with a scintillating fibre target. The measured precisions on trackposition and -direction are $10 \mu \mathrm{m}$ and $5 \mathrm{mrad}$ respectively.

\#6 ISPA-tube prototypes with electrostatic or proximity focussing are also envisaged, depending on the applications considered. 
The ISPA-tube paves the way to a number of applications (particle- and nuclear physics, medicine, biology, astronomy, etc.), with tube designs (proximity-, electrostatic- or magnetic-focussed with one or several chips on the anode) and chip designs (pixel sizes and electronics architectures) adapted to the specific needs of the user.

\section{ACKNOWLEDGEMENTS}

We are indebted to Prof. A. Zichichi for his continuous interest and generous support of our work. We thank E. Heijne and M. Campbell for their advices. We also thank H. Beker, E. Chesi and F. Cindolo for their help in software and hardware, A. Menchikov for re-designing part of the data acquisition electronics and S. Tailhardat for the careful scintillation measurements of the fibre bundles. The invaluable help and advices of J. Dupont and J.-P. Dupraz were particularly appreciated. Finally, we acknowledge the effective work of our industrial partners.

\section{REFERENCES}

[1] T. Gys et al., in "New Technologies for Supercolliders", Proceedings of the 12th Workshop of the INFN ELOISATRON Project, Ettore Maiorana Centre for Scientific Culture, Erice, Italy, September 15-20 1990, Eds L. Cifarelli and T. Ypsilantis, Plenum Press 1991.

[2] C. D'Ambrosio et al., in Proceedings of the "Joint International Lepton-Photon Symposium \& Europhysics conference on High Energy Physics" Geneva, Switzerland 25 July - 1 August 1991, Eds S. Hegarty, K. Potter and E. Quercigh, World Scientific 1992.

[3] C. D'Ambrosio et al., RD-7 Status Report, CERN/DRDC 93-42, 10 Oct. 1993

[4] F. Anghinolfi et al., IEEE Trans. Nucl. Sci. NS-39 (1992) 654.

[5] E. H. M. Heijne et al., CERN/ECP 94-1 (1994), submitted to Nucl. Instr. and Methods in Physics Research.

[6] C. D’Ambrosio et al., NIM A325 (1993) 161.

[7] C. D'Ambrosio et al., NIM A332 (1993) 134.

[8] H. Güsten et al., J. Phys. Chem. 82 (1978) 459.

[9] C. D'Ambrosio et al., NIM A307 (1991) 430.

[10] C. D'Ambrosio et al., NIM A322 (1992) 20.

[11] F. Anselmo et al., NIM in print.

[12] C. D'Ambrosio, in "Supercolliders and Superdetectors", Eds W.A. Barletta and H. Leutz, World Scientific (1993).

[13] C. D'Ambrosio et al., NIM A338 (1994) 389.

[14] C. D'Ambrosio et al., NIM A345 (1994) 279. 


\section{FIGURE CAPTIONS}

Fig. 1 a) Experimental layout. The fibre ribbon is composed of eight fused fibre bundles, each containing 1600 individual scintillating fibres of $60 \mu \mathrm{m}$ diameter. The hodoscope, with its two bulk scintillators of square cross-section (10 $\mathrm{mm} \mathrm{x}$ $10 \mathrm{~mm}$ ), triggers the ISPA-tube readout.

b) Cross-section of fibre ribbon ( $10 \mathrm{~mm} \times 5 \mathrm{~mm}$, full lines) composed of eight square fibre bundles. The active chip surface of $7 \mathrm{~mm} \times 4 \mathrm{~mm}$ is indicated with dashed lines. The upper left-hand region displays the individual fibres $(60 \mu \mathrm{m}$ diameter) and the silicon pixels $(75 \mu \mathrm{m} \times 500 \mu \mathrm{m})$.

Fig. 2 Typical track patterns from cosmic muons. Frame size: $8 \mathrm{~mm} \times 4.8 \mathrm{~mm}$; active size: $7 \mathrm{~mm} \times 4 \mathrm{~mm}$. The firing pixels are clearly seen. Occasional hits sideways of the track are attributed to $\delta$-rays. The spatial precision (standard deviation of the residuals) amounts to $-40 \mu \mathrm{m}$, the track center-of-gravity precision and the angular resolution amount to $\sim 10 \mu \mathrm{m}$ and $\sim 5 \mathrm{mrad}$ respectively.

Fig. 3 Photoelectron spectrum taken with LED flashes. The Poissonian distribution for 4.22 average phoelectrons fits exactly the measured intensities between the pedestal and twelve photoelectrons. 


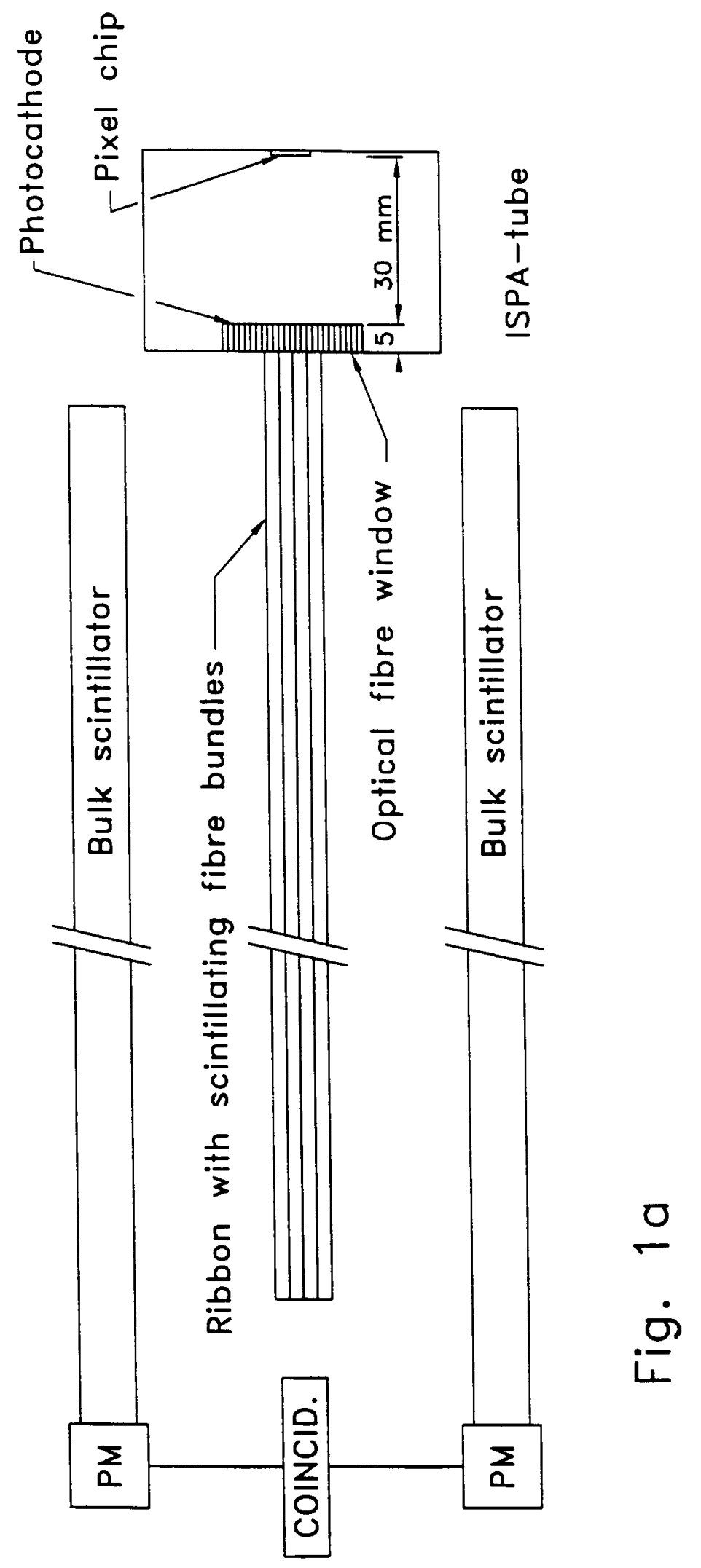




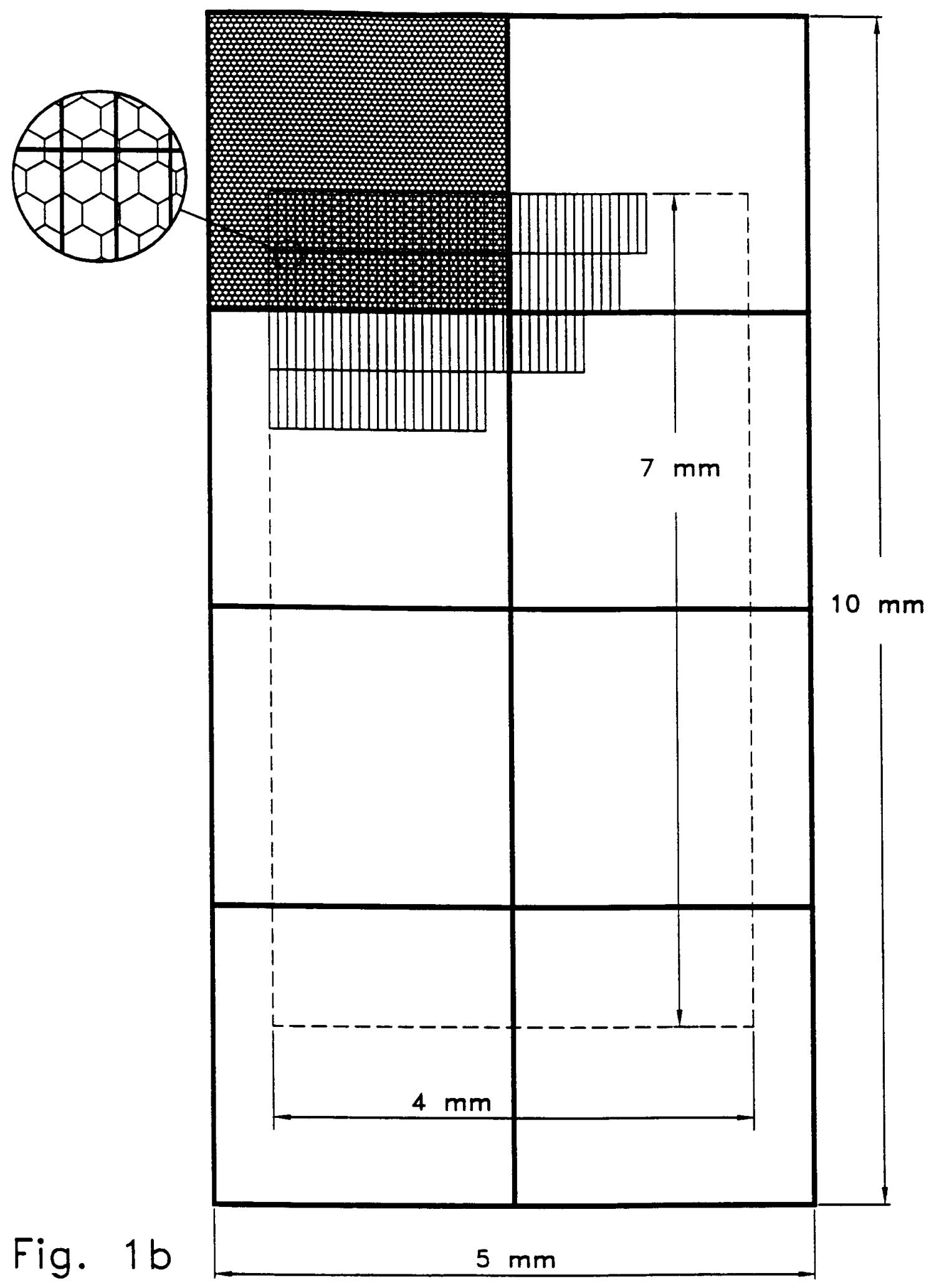




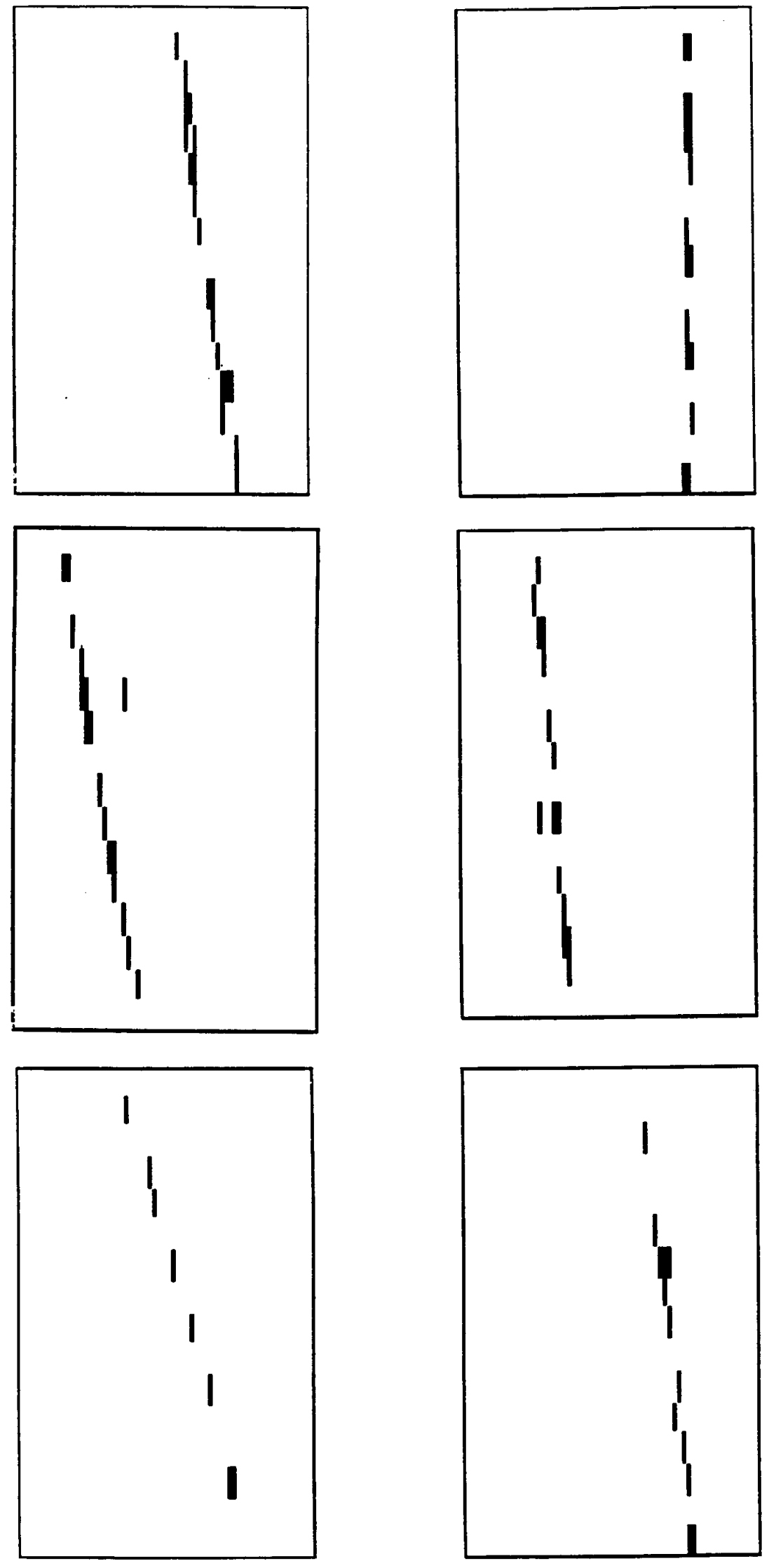

Fig. 2 


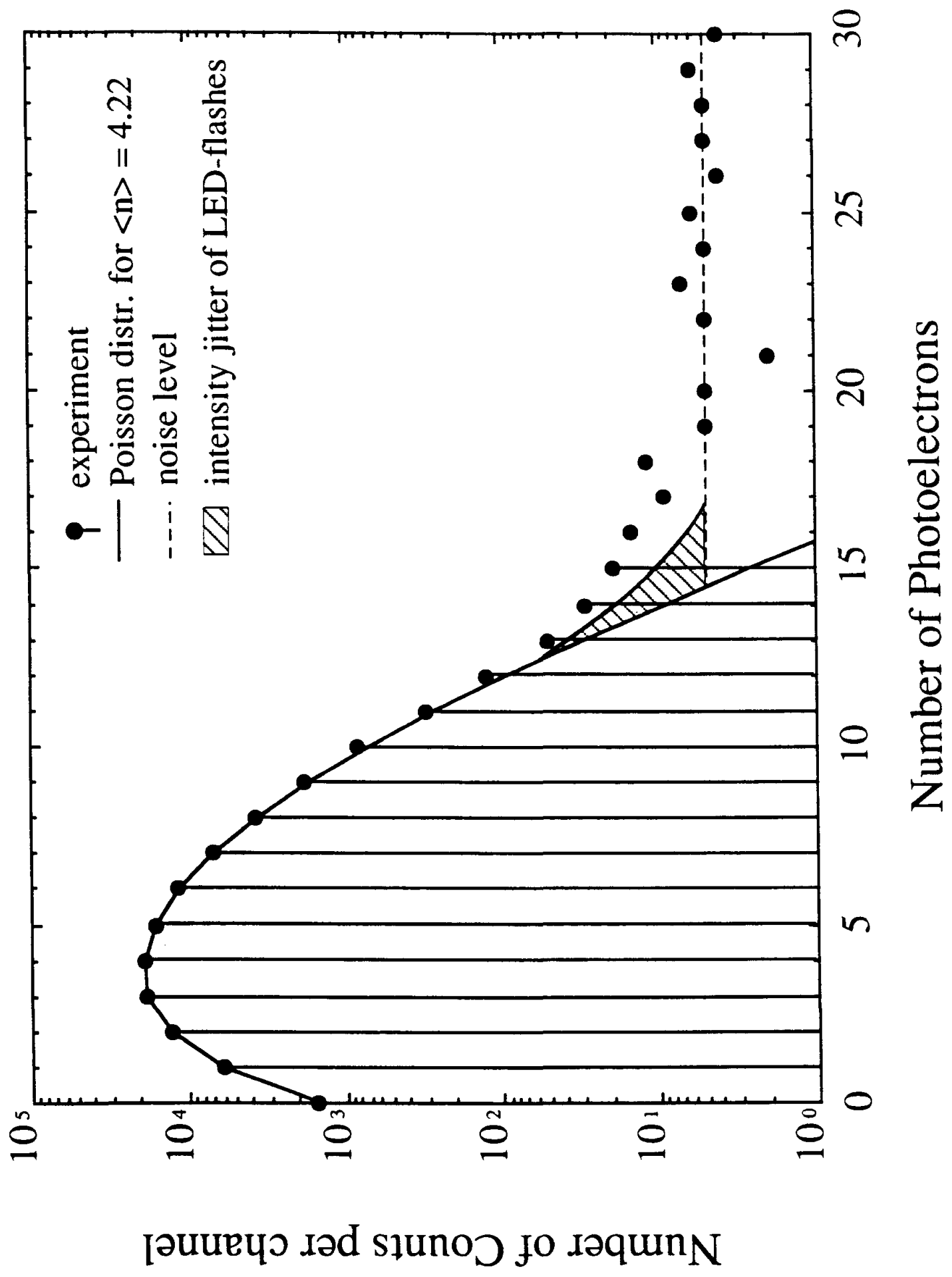

$\stackrel{n}{00}$ 